\title{
Chapter 2 \\ Pseudomonas entomophila: A Versatile Bacterium with Entomopathogenic Properties
}

\author{
Guennaelle Dieppois, Onya Opota, Jorge Lalucat and Bruno Lemaitre
}

\begin{abstract}
Pseudomonas entomophila is unique among Pseudomonas species in being able to activate a systemic immune response in both Drosophila larvae and adults. It has been subsequently shown that oral infections with high doses of this bacterium are highly pathogenic to Drosophila and cause massive destruction of the Drosophila gut epithelium. Besides Drosophila, P. entomophila was able to kill other insects from at least three different orders, suggesting that it has a potentially wide host range and making it a promising model for the study of host pathogen interactions and for the development of bio-control agents against insect pests. In order to unravel the features contributing to $P$. entomophila's pathogenic properties, its complete genome was sequenced and genetic screens were performed to identify virulence factors encoded by this bacterium. The aim of this chapter is to review the current knowledge we have on this bacterium with a particular focus on the pathogenesis it induces, its virulence effectors and their genetic regulation.
\end{abstract}

Keywords Pseudomonas - Drosophila • gut • virulence - NRPS - regulation • entomopathogenic

B. Lemaitre $(\triangle) \cdot$ G. Dieppois

Global Health Institute, EPFL-GHI-SV-UPLEM-, Station 19, CH-1015 Lausanne, Switzerland e-mail: bruno.lemaitre@epfl.ch

O. Opota

Institut de Microbiologie, CHUV, Laboratoire de Diagnostic Moléculaire, 313/IMU/03 Bugnon 48, CH-1011 Lausanne, Switzerland

J. Lalucat

Microbiologia, Departament de Biologia, Edifici Guillem Colom, Universitat de les Illes Balears, Campus UIB, 07122 Palma de Mallorca, Spain

(C) Springer Science+Business Media Dordrecht 2015

J.-L. Ramos et al. (eds.), Pseudomonas, DOI 10.1007/978-94-017-9555-5_2 


\section{An Introduction to P. entomophila}

Pseudomonas entomophila is unique among Pseudomonas species in being able to naturally infect and kill insects upon ingestion. Since it was first identified in 2005, P. entomophila has become one of the most important models for the study of insect-microbe interactions. It was originally isolated from a single Drosophila melanogaster female collected in Calvaire (Guadeloupe) in a screen to identify bacterial pathogens of Drosophila, and was named strain L48T. Upon ingestion, this strain was able to activate a systemic immune response in both Drosophila melanogaster larvae and adults (Vodovar et al. 2005). It has been subsequently shown that oral infections with high doses of this bacterium are highly pathogenic to Drosophila and cause massive destruction of the Drosophila gut epithelium. Since the strain L48T belonged to the Pseudomonas genus and exhibited entomopathogenic properties, it was named Pseudomonas entomophila. Besides Drosophila, P. entomophila was able to kill other insects from at least three different orders, suggesting that it has a potentially wide host range and making it a promising model for the study of host pathogen interactions and for the development of bio-control agents against insect pests. To unravel the features contributing to $P$. entomophila's pathogenic properties, its complete genome was sequenced (Vodovar et al. 2006). In parallel, studies were performed to identify virulence factors encoded by this bacterium. This approach, combined with mechanistic studies on the host immune response and pathology, has provided considerable insights into the interaction of this bacterium with its insect host. The aim of this chapter is to review the current knowledge we have on this bacterium with a particular focus on the pathogenesis it induces, its virulence effectors and their genetic regulation.

\section{Insights from Phenotypic and Genomics Analyses}

\section{Phenotypic and Genomic Features}

The first studies of the entomopathogenic strain $\mathrm{L} 48^{\mathrm{T}}$ showed beyond doubt that it is closely related to the saprophytic soil bacterium P. putida. Recent work has finally allowed it to be designated officially as a novel Pseudomonas species (Mulet et al. 2012). Its closest relative appears to be $P$. mosselii, a poorly characterized species in the P. putida group that is a clinical isolate (Dabboussi et al. 2002).

Phenotypic characterization revealed that $P$. entomophila cells are Gram negative rods that are motile by means of one polar flagellum. This bacterium is strictly aerobic, catalase and oxidase positive. Figure $2.1 \mathrm{P}$. entomophila produces a fluorescent pigment but no pyocianin. Interestingly, the colonies exhibit a strong hemolytic activity on blood agar plates and a significant protease activity Figure 2.1 on skim milk plates that contain casein. It is also positive in the gelatinase test on the API 20NE strips. Although it is able to tolerate a wide range of temperatures 


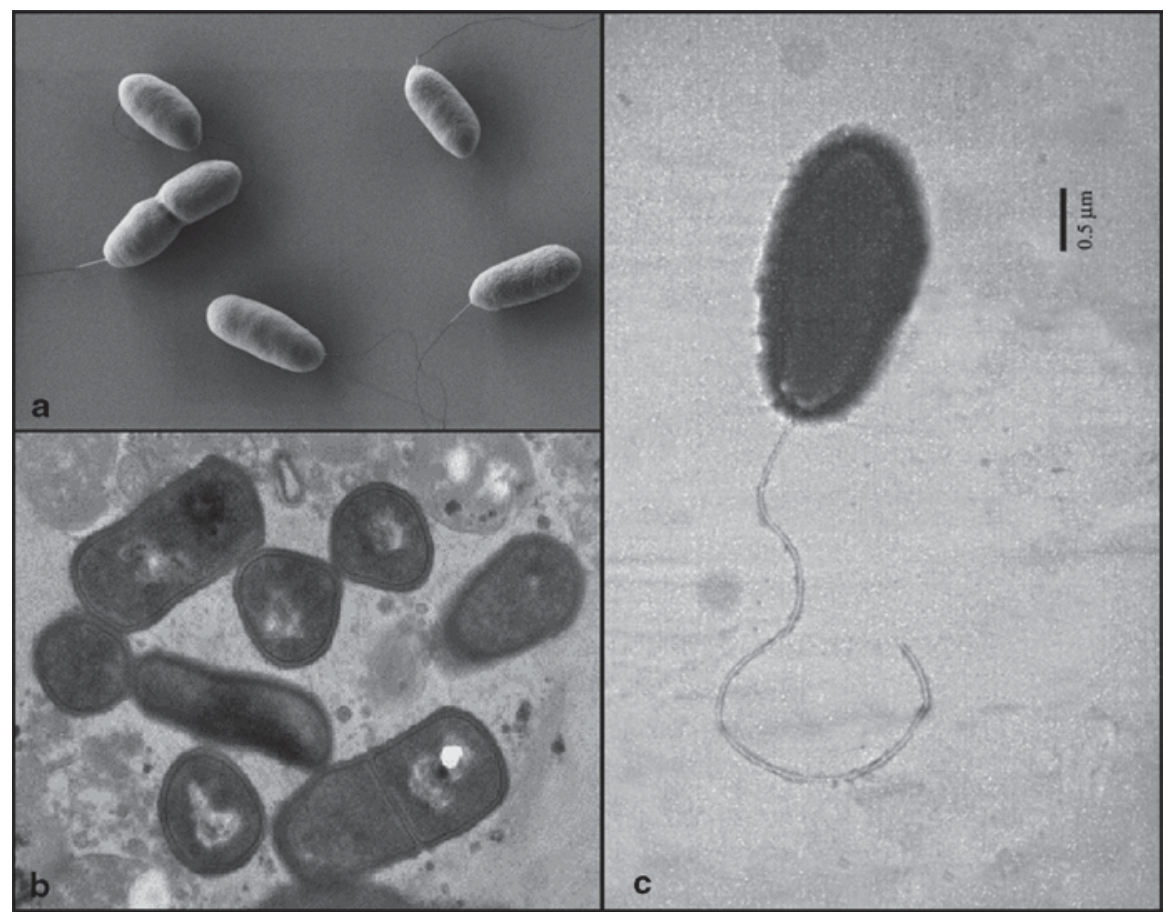

Fig. 2.1 Electron microscopy images of Pseudomonas entomophila L48. a Scanning electron microscopy picture of a Pseudomonas entomophila L48 bacterial culture (EPFL imaging platform B. Lemaitre laboratory). b Transmission electron microscopy picture of Pseudomonas entomophila L48 in the lumen of a Drosophila gut $12 \mathrm{~h}$ after infection (CCME Orsay B. Lemaitre laboratory). c Transmission electron microscopy of a negatively stained cell of P. entomophila L48. (Mulet et al. 2012, Supplementary data)

(4 to $42^{\circ} \mathrm{C}$ ) and $\mathrm{pHs}(\mathrm{pH} 3$ to $\mathrm{pH} 10)$, its optimal temperature growth is around $30^{\circ} \mathrm{C}$ and its optimal $\mathrm{pH}$ between 5 and 9 (Mulet et al. 2012; Guennaëlle Dieppois, unpublished results).

The unique $P$. entomophila chromosome sequence was published in 2006 (Vodovar et al. 2006). It contains 5,888,780 nucleotides with a GC content of $64.2 \%$. It is of an intermediate size compared to the other 20 sequenced Pseudomonas genomes but is smaller than the genome of the opportunistic pathogen P. aeruginosa. Comparisons between the genome of $P$. entomophila and those of five other Pseudomonas species including $P$. putida identified a set of 1002 genes that were unique to $P$. entomophila. In agreement with the close relationship between $P$. entomophila and P. putida, $70.2 \%$ of $P$. entomophila genes have orthologs in $P$. putida. However, the genome of $P$. entomophila has been remodeled to lesser extent by mobile elements such as bacteriophages than that of $P$. putida. This observation could be explained by the fact that the $P$. entomophila genome contains only six putatively active transposase-like proteins. Furthermore, unlike the genome of $P$. putida, the genome of P. entomophila is devoid of type II introns. 


\section{P. entomophila is a Versatile Bacterium}

The complete genome sequence of $P$. entomophila provides interesting insights into this organism's lifestyle. Similar to other Pseudomonas species, the predicted properties of $P$. entomophila indicate that this strain contains a large set of genes involved in the adaptation to multiple carbon sources. This suggests that $P$. entomophila is a metabolically versatile bacterium capable to survive in the soil, rhizosphere and water. Notably, the P. entomophila genome contains several genes that encode proteins with hydrolytic activities such as chitinases, lipases, proteases and uncharacterized hydrolases that might be involved in the degradation of polymers found in the soil. In addition, the $P$. entomophila genome harbors determinants for the catabolism of various aromatic compounds and long-chain carbohydrates making it potentially useful for bioremediation. Finally, P. entomophila encodes more than 535 transporters and a high numbers of regulators suggesting that it is able to adapt to substantial substrate variations. Corroborating these findings, additional P. entomophila strains have recently been isolated from the rhizosphere (Kamala-Kannan et al. 2010) and soil (Shahbaz-Mohammadi and Omidinia 2011) including contaminated soils (Yergeau et al. 2012). However, in contrast to phytopathogenic strains such as $P$. syringae, the genome of $P$. entomophila is devoid of genes encoding enzymes capable of degrading plant cell walls. This is consistent with the observation that this species is not pathogenic for plants (Vodovar et al. 2006). On the contrary, P. entomophila may even benefit plants by promoting resistance to various stresses (Kamala-Kannan et al. 2010). Moreover, Vallet-Gely and colleagues determined that $P$. entomophila was able to protect plants from pathogenic fungi and to promote their growth as $P$. fluorescens does (Vallet-Gely et al. 2010a). This was demonstrated by the ability of cucumber plants to grow in the presence of root pathogenic fungus Pythium ultimum only when $P$. entomophila or alternatively the well-characterized $P$. protegens (formaly fluorescens) biocontrol strain CHA0 was added into the soil.

\section{P. entomophila Iron Acquisition}

Like all other organisms, iron is an essential nutrient for Pseudomonas species. Fluorescent Pseudomonas have adapted to the poor solubility of $\mathrm{Fe}^{3+}$ in their aerobic environment by evolving high affinity iron uptake systems. These systems rely on the uptake of heme or iron siderophore complexes by Ton-B outer membrane receptors that recognize the iron loaded complexes. The $P$. entomophila genome possesses gene clusters encoding proteins required for pyoverdine siderophore biosynthesis and uptake (Vodovar et al. 2006) and for the synthesis of another siderophore related to acinetobactin (Yamamoto et al. 1994). In addition, the P. entomophila genome harbors has $R$ and $h x u C$, the heme uptake-related genes that are typically found in the opportunistic pathogen P. aeruginosa (Ochsner et al. 2000; Bodilis et al. 2009) but not in P. putida. More strikingly, the $P$. entomophila genome carries a considerable number of putative TonB-dependent receptor genes and a high number of 'orphan' TonB-dependent receptor genes that are not found on the 
genomes of P. putida strains (Bodilis et al. 2009; Matthijs et al. 2009). In support of these data, $P$. entomophila is able to utilize a large variety of heterologous pyoverdines siderophores (Matthijs et al. 2009). The wide variety of iron uptake systems in P. entomophila shows that this bacterium is well equipped to survive extreme iron limitation and argues for the importance of iron acquisition in the ecology of this bacterium. This feature may be extremely important not only for its lifestyle in soil but also for its survival within its insect hosts where iron is likely to be rarer. Moreover, for a number of bacterial pathogens including P. aeruginosa, iron deprivation is important in triggering the synthesis of many virulence factors (Prince et al. 1993). In agreement with an essential role of $P$. entomophila iron uptake systems in the infection process, we have recently identified a Ton B receptor gene as an important factor for the virulence of this bacterium (G. D. unpublished data).

\section{P. entomophila as a Source of Secondary Metabolites}

Among the factors that may be fundamental for interaction with insects, the genome of $P$. entomophila harbors five gene clusters associated with production of secondary metabolites. One gene cluster, coding for a non-ribosomal peptidesynthase (NRPS) is responsible for hydrogen cyanide $(\mathrm{HCN})$ production. The other four clusters code for NRPSs predicted to synthetize at least three different lipopeptides and a polyketide of unknown function (Vodovar et al. 2006).

It has indeed been confirmed that $P$. entomophila can synthesize HCN, placing it among the small number of cyanogenic bacteria (Ryall et al. 2009). Although HCN production by $P$. entomophila is regulated by oxygen availability, it does not seem contribute to the pathogenicity towards Drosophila (Vallet-Gely et al. 2010b). The role of the other NRPS gene clusters in P. entomophila virulence has also been investigated (Vallet-Gely et al. 2010b). Only one of them (pseen 0131, pseen0132, pseen 0133,) appears to play a crucial role. It has subsequently been named PVF for $P$ seudomonas Virulence $F$ actor (See below for further details on PVF).

The nature of a product that is synthetized by another NRPS (pseen3332, pseen 3044, pseen 3045 ) has also been deciphered. It is involved in the production of a new cyclic lipopeptide of 14 amino acids and $3 \mathrm{C} 10-\mathrm{OH}$ fatty acids. This lipopeptide is responsible for both the hemolytic and surfactant activity observed in the supernatant of $P$. entomophila and has, thus, been called Entolysin (Vallet-Gely et al. 2010a) (see later).

Although, $\mathrm{HCN}$ and most of the secondary metabolites encoded by P. entomophila do not seem to be essential for its virulence, their production may have other substantial roles. For example in other Pseudomonas species, these molecules are involved in bio-control (Haas 2005), in the killing of nematodes and in suppressing microbial competitors in the soil (de Bruijn et al. 2007; Li et al. 2013; Neidig et al. 2011). Of note, it has recently been observed that $P$. entomophila is clearly pathogenic to C. elegans (Olivier Zugasti personal communication) and was able to outcompete several other species, such as Mycobacterium marinum and the fungus Candida albicans (Onya Opota personal communication). 


\section{Putative Virulence Factors Against Insects}

Several genes in the genome of $P$. entomophila have been associated with its entomopathogenicity. For example, the presence of genes that encode for TccC-type insecticidal toxin are particularly striking since they are only found in entomopathogenic bacteria such as Photorhabus luminescens and Xenorhabus nematophila (Hinchliffe et al. 2010) and absent from other Pseudomonas genomes (Vodovar et al. 2006). Moreover, like $P$. syringae, the $P$. entomophila genome encodes other proteins more distantly related to $\mathrm{Tcc} C$ and $\mathrm{TcdB}$-type insecticidal proteins. The function of these proteins in the virulence towards insect has not yet been tested.

Proteases are also thought to contribute to the virulence of bacterial species. Interestingly, P. entomophila encodes three serine proteases (pseen 3027 , pseen 3028 , pseen 4433) and one alkaline protease (pseen 1550) absent from P. putida. The latter is a homolog of AprA which has been shown to be involved in virulence in other bacteria by protecting against the immune response and degrading of host tissues (Hong and Ghebrehiwet 1992; Leduc et al. 2007; Miyoshi and Shinoda 2000; Parmely et al. 1990; Travis et al. 1995). Notably, AprA has been shown to be the most abundant protein in P. entomophila supernatant (Liehl et al. 2006). Illustrating its importance, the visible protease activity of $P$. entomophila on skim milk plates is completely abolished in an aprA mutant. How AprA contributes to P. entomophila pathogenesis will be more specifically addressed in another section of this chapter.

P. entomophila also carries a number of genes coding for cell surface associated factors that usually contribute to pathogenesis by allowing adhesion to the host surface and thus, an effective colonization. Among them, we find genes coding for filamentous haemagglutinin, a surface adhesion protein and the amyloid curli fiber. In agreement with a role of these genes in P. entomophila virulence, they often cluster with genes coding for type I secretion systems (T1SS). T1SS, T3SS, T4SS, and T6SS are actively involved in the virulence of pathogenic bacteria as they promote the direct delivery of exo-proteins into the extracellular medium (T1SS) or into the host cell (T3SS, T4SS, and T6SS).

Interestingly, P. entomophila is the first Pseudomonas strain that is pathogenic in a multicellular organism and yet is devoid of a T3SS secretion system. This bacterium only possesses a single locus containing the conserved core genes of T6SS proteins as well as several T6SS homolog proteins (VgrG and Hcp) dispersed in the genome. Notably, we found that components of the T6SS (Vgr, Rhs and Hcp proteins) were among the most abundant proteins excreted in the supernatant of this bacterium (Opota et al. 2011). Moreover, this system might actively participate in P. entomophila virulence since T6SS mutants were found in a screen for factors contributing to $P$. entomophila pathogenesis (G. D. unpublished). Owing to the lack of the common T3SS and T4SS, the single T6SS could play an essential role in the insect-bacterium interactions (Sarris and Scoulica 2011).

Overall, the analysis of the P. entomophila genome and its general phenotypic properties indicate that this bacterium has the potential to infect and colonize insect niches. How this is useful for the bacteria's life cycle remains unclear, as it 
has never been found associated with other insects in the wild beyond its initial isolation. However, the use of this bacterium as a model to study insect pathogen interaction has helped to understand the interconnected nature of the mechanisms underlying pathogenesis and the host immune pathways.

P. entomophila has so far been shown to be pathogenic for three orders of insects from Diptera (Anopheles gambiae D. melanogaster), Lepidoptera (e.g. Bombyx mori, Galleria mellonella) and Coleoptera (e.g. Sitophilus oryzae). It is also lethal to the ameobae Dictyostelium discoideum (Vallet-Gely et al. 2010a) and leads to the slow killing of C.elegans (O Zugasti personal communication). P. entomophila pathogenesis has been mostly studied in Drosophila adults and larvae. In a model of septic injury (i.e. direct pricking of a culture pellet into the body cavity), this pathogen kills flies in a day. When $P$. entomophila is ingested at high doses by adults $\left(\mathrm{OD}_{600} 50-100\right)$, the flies succumb to infection within 2-3 days. Survival

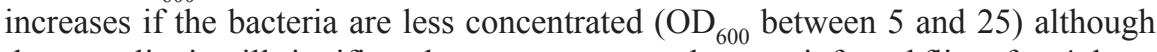
the mortality is still significantly greater compared to non-infected flies after 4 days. However, below $\mathrm{OD}_{600} 1$ ingestion of $P$. entomophila is apparently not lethal for the flies (Buchon et al. 2009a) indicating that only a large dose can overcome the Drosophila immune system and kill flies (Figs. 2.1 and 2.2). Of note, none of the other Pseudomonas species exhibit the same level of virulence towards Drosophila when ingested at high dose, demonstrating specific pathogenic properties of $P$. entomophila. Considering these observations, the overall pathogenesis of $P$. entomophila must depend on the following properties (i) the ability to enter and persist in the gut which is related to its capacity to survive both physicochemical conditions and the immune defenses of this organ (ii) the excretion of toxic substances that disrupt the host physiology.

\section{Survival of P. entomophila in the Harsh Conditions of the Drosophila Gut}

The mechanisms by which ingestion of high doses of $P$. entomophila can kill Drosophila have been well characterized. We have observed that after starvation Drosophila does not particularly avoid $P$. entomophila and ingests high doses easily when the bacteria pellet is mixed with sucrose (G.D. unpublished). Similar conditions might occur in the wild since bacteria are found at high concentrations in decaying fruits.

Interestingly one of the most overt phenotype observed after the ingestion of relevant doses of $P$. entomophila is an immediate blockage of food uptake (Liehl et al. 2006; Vodovar et al. 2005). This results in a visible accumulation of ingested bacteria in the fly crop. This food-uptake blockage phenotype is also associated with other entomopathogenic bacteria such as Serratia entomophila and Yersinia pestis (Vallet-Gely et al. 2008). How this phenomenon occurs and contributes to pathogenesis is still unclear. It has not yet been established whether P. entomophila directly induces this phenomenon or if food uptake blockage is a host response to intestinal damage associated with the infection process. 

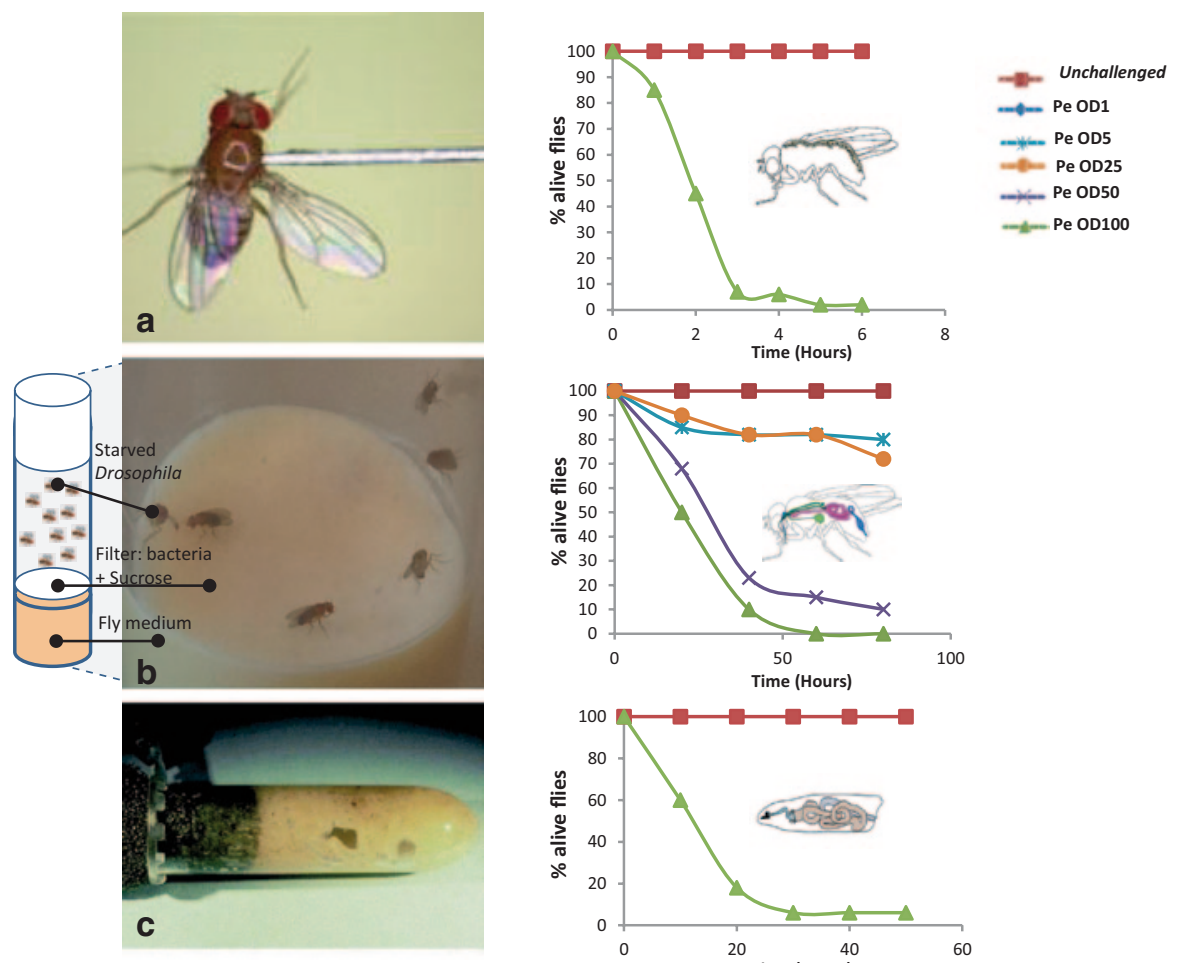

Fig. 2.2 Methods developed to infect Drosophila with P. entomophila. a Systemic infection. Bacterial injection is achieved by pricking adult flies in the thorax with a needle that has been dipped into a concentrated bacterial solution or by microinjecting a precise dose of microbes into the body cavity. Through this type of infection, all microbes induce a strong immune response that is specific for that type of microbe as they are in direct contact with immune system sensors in the body cavity. P. entomophila kills flies within a few hours only. b Natural infection of Drosophila adults. To mimic a natural infection by feeding, the adult flies, preferentially females of 3-5 days are starved prior to the infection. They are then transferred to in fly vials containing $200 \mathrm{ul}$ of a bacterial pellet usually at $\mathrm{OD}_{600} 100$ mixed with $1 \%$ sucrose put on a filter on top of the fly medium in the vial. The fly medium contains antibiotics that rapidly inactivate bacteria. Flies are thus infected by a single high dose of live bacteria. After ingestion, the flies die within $2-3$ days with a pellet at $\mathrm{OD}_{600} 100$. They survive longer when the pellet is less concentrated. Their survival is not affected when they ingest bacteria at $\mathrm{OD}_{600}$ below $5\left(5.10^{9}\right.$ cells $\left./ \mathrm{ml}\right)$. c Natural infection of Drosophila larvae. Third instar larvae are mixed with a solution of crushed banana containing bacteria at $\mathrm{OD}_{600}$ 100 for one hour before being transferred back to a normal fly vial. Larvae are more susceptible than adults to $P$. entomophila infection and are killed in 1 day. These two types of natural infection are inducing the local immune response in the gut and the systemic immune response. Only few strains are able to induce with these types of infection immune response reflecting that it can efficiently mimic the specifics interactions between Drosophila and its pathogens

However, living and dividing $P$. entomophila cells have been observed by both fluorescence and electron microscopy in the lumen in different regions of the gut, confirming that this bacterium is able to travel along the gut and to resists the gut conditions, at least for $16 \mathrm{~h}$. 
The Drosophila midgut is considered a hostile environment for most ingested bacteria due to its very low $\mathrm{pH}(\mathrm{pH}=2-3$ in the middle midgut) (Shanbhag and Tripathi 2009) and the production of a large number of lysozymes (Daffre et al. 1994). Nevertheless, in vitro studies indicate that $P$. entomophila can grow even at a low pH of 3 (Mulet et al. 2012) and is highly resistant to lysozymes activity ( $2 \mathrm{mg} / \mathrm{ml}$ ) (Opota and Dieppois, personal communication). Interestingly, P. entomophila is also resistant to high concentrations of $\mathrm{H}_{2} \mathrm{O}_{2}$ in vitro (up to $1 \mathrm{mM}$; Dieppois, unpublished). These observations could explain why $P$. entomophila is able to resist those first line defenses, including the microbicidal activities of reactive oxygen species that are immediately produced by in the gut by the NADPH Duox, in response to microbial infections (Chakrabarti et al. 2012; Ha et al. 2005a, b, 2009a, b).

\section{P. entomophila Counteracts the Production of Antimicrobial Peptides}

Another important line of defense is the production of antimicrobial peptides such as Diptericin, Attacin and Drosocin. They are secreted by the gut epithelium upon oral infection gram-negative bacteria. This response is mediated by the Imd signaling pathway which is activated by diaminopimelic acid (DAP)-type peptidoglycan found in bacilli and gram-negative bacteria (Kaneko et al. 2004; Leulier et al. 2003; Stenbak et al. 2004). A microarray analysis showed that P. entomophila infection strongly stimulates the expression of antimicrobial peptide genes in the gut (Chakrabarti et al. 2012). Thus, this bacterium is specifically recognized by the local immune system. Moreover, our laboratory previously showed that overexpression of the Imd pathway in the gut prior to infection confers resistance to $P$. entomophila (Liehl et al. 2006) (Chakrabarti, unpublished results), indicating that this bacterium is to some extent sensitive to the activity of antimicrobial peptides. We recently understood that the ability of $P$. entomophila to survive the Drosophila innate immune response is dependent on its capacity to inflict damages to the gut epithelium.

Histological studies on larvae and adults show that $P$. entomophila infection is associated with epithelial cell damage such as enterocytes displaying abnormal microvilli or undergoing cell death. Electron microscopy pictures show that the peritrophic matrix (PM), a chitinous matrix that lines the midgut epithelium of insect is still present after $12 \mathrm{~h}$ of infection, suggesting that $P$. entomophila is unable to cross this protective barrier and resides within the lumen at least in the first hours of infection (Kuraishi et al. 2011; Vodovar et al. 2005). Consistent with these observations, P. entomophila is not detected in the haemocoel of flies. Hence, this bacterium is able to damage the gut without physically contacting the gut epithelial layer. The severity of intestinal damage is consistent with data showing that the repair and stress pathways are strongly activated upon $P$. entomophila ingestion (Chakrabarti et al. 2012).

It has been shown that $P$. entomophila survives the activation of the immune response because the infection rapidly reduces the translation level in the gut 
epithelium cells as an adaptive stress response to damages inflicted by the bacterium (Chakrabarti et al. 2012). While antibacterial genes are induced at the transcriptional level in the gut upon infection with $P$. entomophila, the effective production of antimicrobial peptides is blocked due to this general inhibition of translation that affects the translation of all newly synthesized transcripts.

\section{P. entomophila Ingestion Leads to Death by Rupture of the Gut}

Another consequence of this translational arrest is the inhibition of the epithelium renewal program which is necessary to repair the damage caused by the infection (Amcheslavsky et al. 2009; Buchon et al. 2009b). Studies using non-lethal bacteria pathogens have shown that recovery from infection only occurs when bacterial clearance by the immune system is coordinated with a repair of tissue damages. The current model is that translational inhibition is the primary cause of fly death by preventing both immune and repair pathways. As a consequence, the bacteria are not eliminated and flies succumb as their gut shrink and rupture.

The reduction of translation is a direct consequence of the excessive activation of stress pathways caused by the cellular damage inflicted to the intestine by $P$. entomophila. Two factors contribute to these cellular damages. P. entomophila produces toxins that cause epithelium damage (Opota et al. 2011). However, the main factors damaging the gut epithelium are the reactive oxygen species (ROS) produced by the host itself. Several studies in Drosophila have demonstrated the importance of intestinal ROS production surviving oral infection (Ha et al. 2005b, 2009b). However, the level of ROS produced by the gut is significantly higher upon P. entomophila ingestion than with other pathogenic but non-lethal bacteria such as Erwinia carrotovora subsp. Carrotovora strain 15 (Chakrabarti et al. 2012). In the case of $P$. entomophila infection, this oxidative burst not only fails to eliminate the bacterium but is also highly deleterious to the gut. By both creating irreversible damages and inducing the translational inhibition that prevents repair and immune response, the high level of ROS that results from ingesting $P$. entomophila is the cornerstone of this bacterium's pathogenesis.

\section{The Toxins of $P$. entomophila}

As mentioned above, the $P$. entomophila genome contains a large set of genes encoding putative virulence factors (Vodovar et al. 2006). A genome wide transposon mutagenesis subsequently led to the identification of some of the genes involved in virulence. With the exception of the genes coding for the NRPS synthesizing PVF, no genes predicted to be virulence effectors were identified in the initial screens (Vallet-Gely et al. 2010b; Vodovar et al. 2006). In contrast, mutations in several genes encoding regulators significantly attenuated virulence. Furthermore, it later turned out that PVF was also a regulatory factor instead of 


\section{严 Springer}

http://www.springer.com/978-94-017-9554-8

\section{Pseudomonas}

Volume 7: New Aspects of Pseudomonas Biology

Ramos, J. -L.; Goldberg, J.B.; Filloux, A (Eds.)

2015, XII, 316 p. 40 illus. in color., Hardcover

ISBN: 978-94-017-9554-8 